MATEC Web of Conferences 44, 01002 (2016)

DOI: $10.1051 /$ matecconf/20164401002

(C) Owned by the authors, published by EDP Sciences, 2016

\title{
MQAM Modulation Recognition Based on AP Clustering Method ${ }^{1}$
}

\author{
Qiang $\mathrm{Li}^{1, \mathrm{a}}$, Dong Shen ${ }^{1}$ and Fei Wang ${ }^{1}$ \\ ${ }^{1}$ School of Electronic and Information Engineering, Lanzhou Jiaotong University, Lanzhou, China
}

\begin{abstract}
For signal analysis in the management and monitoring of the radio spectrum, it is need to automatically identify the modulated signal. This paper performs clustering algorithm on signal constellation diagram after equalization through AP clustering method, and proposes a clustering method for limited problem of modulation recognition type. After normalizing the shape of the constellation diagram, the size of the constellation diagram, and signal AP clustering, it realizes the matching recognition of the constellation diagram. The simulation results show that the AP clustering has efficient recognition ability.
\end{abstract}

\section{Introduction}

With the rapid development of modern communication technology, in the civil, modulation recognition of communication signals is mainly applied to software radio [1], spectrum monitoring and management, cognitive radio and so on [2-3]. In the increasingly complicated signal environment, some of the illegal users interfere and utilize the wireless spectrum, affecting the normal communication of legitimate users seriously. Spectrum resources management departments need to detect the interference of illegal radio and identify the modulation type of the interference signal, so as to having better monitoring and management of radio resources. For achieving all of these, modulation identification technology must be used.

In the military, electronic warfare has become the main form in the future. The battlefield information mainly is transmitted by radio, so the communication signal detection has become the important content of electronic warfare, which is an important premise to implement the communication countermeasure [4-6]. Communication signal detection firstly is to monitor the enemy signal, and using the receiver to intercept the enemy signal, and then the intercepted signal will be analyzed and processed, obtaining the communication signal modulation type and finally the useful information. Modulation recognition technology is helpful in choosing the optimal jamming technology in electronic warfare. While taking effective suppression and destruction to enemy signals, the communication of friendly parts will be ensured. In addition, the modulation recognition technology can also be used for radiating source recognition [7]. According to the modulation type of the received signal and some other measurement parameters, the type of enemy detector will be detected, distinguishing the kind of radar signal and analyzing the threat level, so as to improve the survival ability of the platform.

In recent years, with the rapid development of digital modulation techniques, the modulation method of communication signals are more complicated and diversity. M-ary quadrature amplitude modulation is a

\footnotetext{
${ }^{\text {a }}$ Corresponding author: Liqiang_fly@163.com
} 
kind of commonly used digital modulation mode of modern wireless communication system which is widely used in satellite communication, network communication, microwave communication and cable TV networks. At the same time, the MQAM has also become the modulation way for broadband access network technology-ADSL, VADSL and modern CATV network. Comparing with other modulation techniques, MQAM modulation technology has the characteristics of flexible modulation modes, strong anti-noise performance and so on. In addition, MQAM is especially suitable for occasions of limited spectrum resources [8]. So researching on MQAM modulation mode recognition technology has very important application value.

There are two main methods of signal modulation recognition, one is inter-class recognition, such as the recognition between MPSK and MQAM; another is within-class recognition that is different modulation order recognition to the same signal, such as between different hexadecimal identification of MQAM [9]. This paper focuses on the inter-class recognition which is based on constellation diagram modulation type, the premise is: if a digital modulation signal can be only expressed by its constellation diagram, then it can also be identified by restoring the constellation diagram at the receiver.

\section{Signal model}

Given the signal received by the receiver is:

$$
r(t)=\operatorname{Re}[s(t)+n(t)]
$$

where $s(t)$ is modulation signal, the expression is:

$$
s(t)=\operatorname{Re}\left[\sum_{n} A_{k} g\left(t-n T_{s}\right) \cos \left(2 \pi f_{c} t+\varphi_{n}\right)+n(t)\right] 0<t<\mathrm{T}=\mathrm{NT}_{s}
$$

$A_{k}$ is the amplitude of the kth symbol, $T_{S}$ is the symbol width, $f_{c}$ is the carrier frequency, $\varphi_{n}$ is the phase error, $N$ is the receiving characters in $T, g\left(t-n T_{s}\right)$ is the baseband pulse, $n(t)$ is the white Gaussian noise.

\subsection{Constellation graphical shape normalization}

In order to make the constellation diagram got fits to the constellation diagram model in the database, constellation diagramical shape must be normalized. The center of the constellation diagram can be obtained by the mean of all cluster centers. In order to calculate conveniently, the constellation diagram center can be takes as the origin of coordinate. Due to the angle $\theta$ of phase error is a random variable, so the position relationship between the clustering centers obtained can judge if the constellation has deflection or not. If there is a deflection, then the deflection angle is estimated, further more the standard form constellation diagram can be got with the constellation diagram rotating reversely by the same angle.

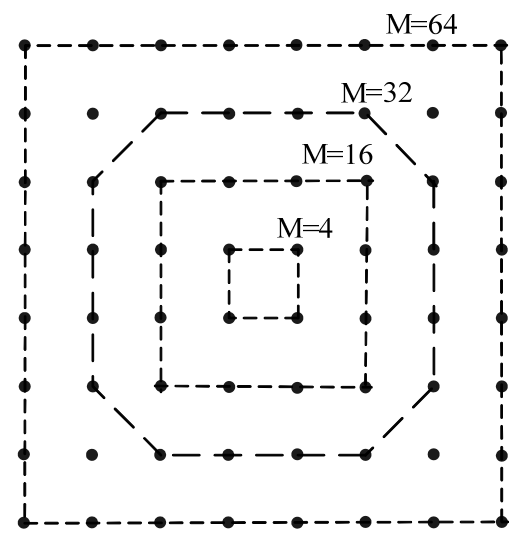

Figure 1. Constellation diagram of rectangular MQAM modulation signal

As shown in Fig.1, in the constellation diagram of the rectangular MQAM, regardless of how much the value of $\mathrm{M}$ is, the constellation points for $M=4$ are fixed. According to the distribution of these four points, the rotation angle of the signal constellation diagram can be obtained. The specific approach is: in the standard signal constellation diagram, the minimum positive angle value in the four points is $\pi / 4$. The minimum positive angle of four points having smaller radius in all the points in constellation diagram minus $\pi / 4$, the obtained minimum positive value is the reverse rotation angle. Considering the distribution of the information carried on the periphery of the point symbol energy greater than the inner point of the information carried by the symbol of energy, that is in the same SNR condition, influence of the interference at the periphery points is relatively small. So for high order MQAM signal, using peripheral vertex information as far as possible, the influence of noise will be reduced. The above steps achieve the constellation 
reverse rotation which may not be synchronized in the carrier phase, namely the constellation graphical shape normalization.

\subsection{Constellation graphical size normalization}

AP clustering will get the constellation diagram in the [-1, 1] plane. In order to match with the model in constellation library, the constellation diagram needs to be mapped to the $64 \times 64$ plane. As long as adjusting constellation diagram abscissa and ordinate respectively, constellation diagram size normalization can be realized [10]. Given the adjusting parameters of abscissa is $m u l_{-} x$, adjusting parameters of ordinate is $m u l_{-} y$, $m u l_{-}$ratio is $m u l_{-}$ratio $=\frac{m u l_{-} x}{m u l_{-} y}$. For symmetric constellation diagram, the value of mul_ratio is 1 .

$$
m u l_{-} x=m u l_{-} y=\frac{k}{\max (y)-\min (y)}
$$

$\max (y)$ represents the maximum value, $\min (y)$ represents the minimum value. The $\mathrm{K}$ value has the relationship with the structure of the histogram, each cluster center is moved to the plane center of size $16 \times 16$. With 16QAM, $\mathrm{K}$ is 48 , for different $\mathrm{M}$, the value of $\mathrm{K}$ is also different, specifics seen in reference [11].

In order to realize constellation diagram model, the shape of signal constellation diagram must be represented discretely. So we need to mesh the constellation diagram plane, the size of meshes affects the amount and accuracy of calculation. If the meshes are too large, the recognition accuracy will decline. The meshes are too small, the amount of calculation for the algorithm is great. The following method is to use Parzen window to grid [12].

Still with 16QAM signal as an example, Constellation diagram is composed of 16 vertices generated by the clustering module. In order to easy representation, the whole constellation diagram is mapped to a discrete grid with size $64 \times 64$, each modulation state is a pixel of image with size $64 \times 64$. The initial size $V_{0}$ of resolution unit should be able to meet enough detail to obtain the probability density function of the constellation diagram shape.

$$
V_{0} \leq \frac{64^{2}}{M}
$$

where $M$ is the constellation diagram modulation state.
For the 16QAM signal constellation diagram in grid with size $64 \times 64$, the initial $V_{0}$ of the kernel function base is the unit size $64 \times 64$. Obtained from the Parzen window method, each resolution unit must be further divided to obtain the details of each vertex distribution. When 4096 data points from each constellation, i.e. each vertex has 256 data points, the kernel function base [13]:

$$
\rho=\frac{V_{0}}{\sqrt{n}} \quad n=256
$$

Therefore, for each vertex of the density function to obtain the Parzen window is a unit with size $4 \times 4$. At the same time, the Parzen window of 4QAM signal is a unit with size $2 \times 2$, the Parzen window of 64 QAM signal is a unit with size $8 \times 8$.

\section{Constellation diagram matching}

Taking the constellation diagram matching as the classification process, according to the clustering results, the points of constellation diagram in choosing modulation set are assigned to each class, the specific method is:

Based on Euclidean distance, the similarity matrix $s(i, j)$ of these data points is defined as:

$$
s(i, j)=-\left\|\left(x_{i}-x_{j}\right)^{2}+\left(y_{i}-y_{j}\right)^{2}\right\|
$$

All the data points in original sample set are potentially paradigm samples, so all the $s(m, m)$ initialization is the same. In the clustering process, there are two variables, the degree of attraction $r(i, j)$ and the degree of membership $a(i, j)$, which need to be updated by the competition mechanism. The update rules are:

$$
\begin{gathered}
r(i, j)=s(i, j)-\max _{j^{\prime} \text { s.t.j.j' } \neq j}\left\{a\left(i, j^{\prime}\right)+s\left(i, j^{\prime}\right)\right\} \\
r(j, j)=s(j, j)-\max _{j^{\prime} \text { s.t.j.j }} \neq j \\
a(i, j) \\
=\min \left\{0, r(j, j)+\sum_{i^{\prime} \text { s.t. } i^{\prime} \neq i, j} \max \left\{0, r\left(i^{\prime}, j\right)\right\}\right\} \\
a(j, j)=\sum_{i^{\prime} \text { s.t. } i^{\prime} \neq j} \max \left\{0, r\left(i^{\prime}, j\right)\right\}
\end{gathered}
$$

By the updating rules above [14], the information is exchanged between the sample points, finally the function chosen achieves minimum. The 
AP algorithm can be terminated by a fixed number of iterations or changing extent in the local. The symbol numbers here $N=400$.

The specific algorithm flowchart is:

To compute the similarity between the $N=400$ points values, the value is placed in the similarity matrix $s(i, j)$, where $s$ is $N \times N$ matrix.

Diagonal value of the similarity matrix $S$ is selected as the value of the $P$ parameter. After the iterative process begins, the $\mathrm{R}$ value and a value are computed at each time.

With the value of $r(j, j)+a(j, j)$ to determine whether is the clustering center or not, the calculation will be terminated when the clustering center value do not change after 100 times iteration.

\section{Simulation analysis}

Under the hypothesis of not account for blind equalization and after constellation graphical shape normalization, from the AP algorithm above, the number of symbols is $N=400$, the number of iterations is 100 , the reconstruction figure of the rectangular 16QAM constellation diagram are shown as follows. Fig.2 and Fig.3 represent the performance of AP algorithm respectively under different SNR. From the figures below, it can be seen that AP clustering can basically identify the positions of constellation points when $\mathrm{SNR}=12$. When $\mathrm{SNR}=18$, AP clustering can accurately identify the positions of constellation points.

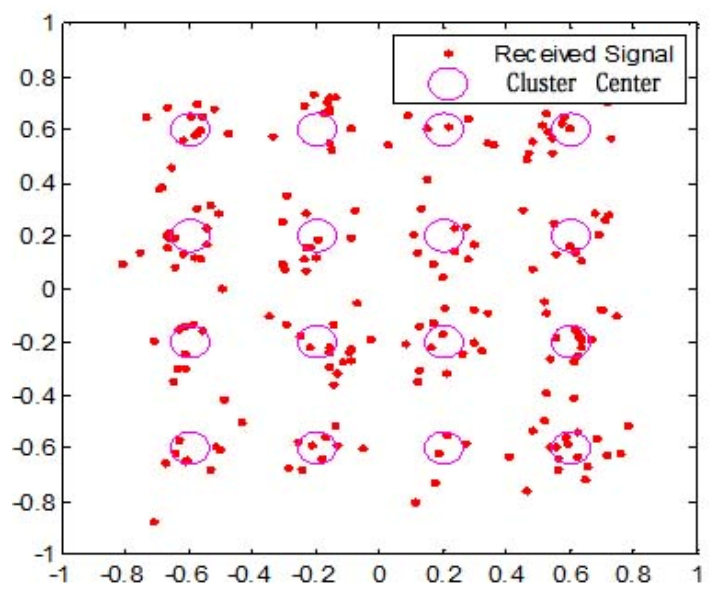

Figure 2. 16 QAM clustering when $\mathrm{SNR}=12$

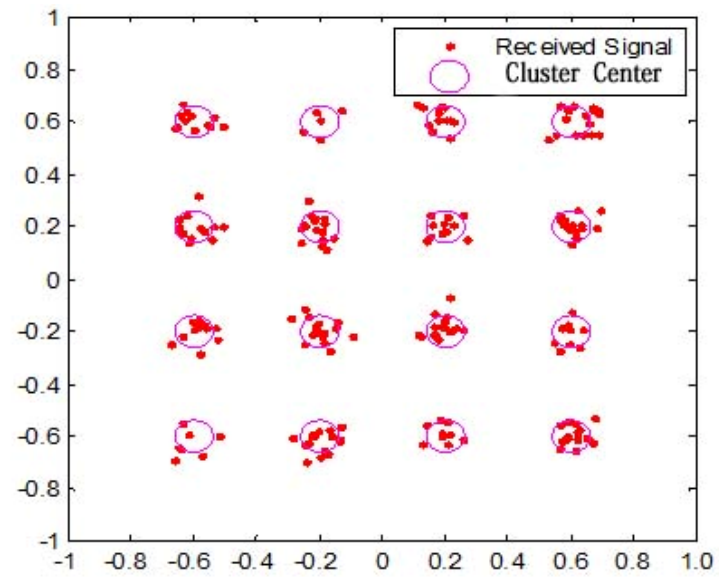

Figure 3. 16 QAM clustering when $\mathrm{SNR}=18$

Fig.4 is the recognition success rate of QAM signal from $\mathrm{M}$ hexadecimal modulation, the symbol length is 400 , it can be seen that AP clustering recognition rate has reached $90 \%$ when the $\mathrm{SNR}=12$. But the recognition rate curve is nonlinear, modulation recognition has the threshold effect. If the ideal results need to be got, the requirements for the SNR should be more than 12 .

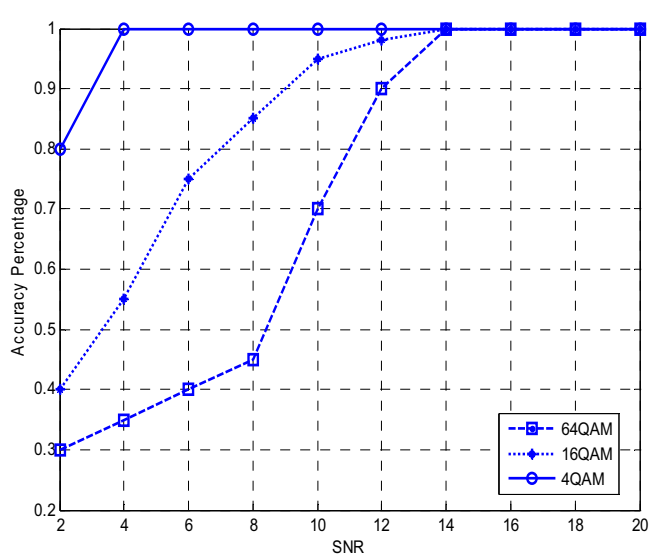

Figure 4. the recognition success rate of QAM signal from M hexadecimal modulation (the symbol length is 400)

\section{Conclusions}

This paper mainly studies the recognition of MQAM signals which has important application value in modern communications. The method of AP clustering reconstruction constellation diagram matching is used here to recognize the MQAM signals, cluster center is extracted by the constellation diagram clustering which is got by constellation graphical shape normalization by using 
the clustering methods, and then identification is used here. The simulation results show that the algorithm can basically identify the positions of modulation signals when $\mathrm{SNR}=12$. For different modulation mode from $\mathrm{M}$ value, the modulation recognition success rate has reached $90 \%$ when the $\mathrm{SNR}=12$. The modulation recognition success rate is greater than $99 \%$ when $\mathrm{SNR}=16$

The method of AP cluster reconstruction constellation diagram matching has good practicability for MQAM signals recognition.

\section{Acknowledgement}

This work was supported by Gansu Natural Science Foundation (No.148RJZA033) and Youth Foundation of Lanzhou Jiaotong University with grant No. 2012002.

\section{References}

1. P. Pinto, S. Silva, H. Miranda, An adaptive software radio receiver architecture for linear bidimensional modulations, The Sixth Baiona Workshop on Signal Processing in Communications, Baiona, Spain, 2003, pp. 211-216.

2. L. C. Freitas, C. Cardoso, F. C. Muller, et al, Automatic modulation classification forcognitive radio systems: Results for the symbol and wave domains, 2009 IEEE Latin-American Conference on Communications, Brazil, Sep. 2009, pp. 1-6.

3. B. Ramkumar, Automatic modulation classification for cognitive radios using cyclicfeature detection, IEEE Circuits and systems Magazine, 2 (2009).

4. D. P. Zhang, Y. F. Chen, X. F. Li, QAM modulation recognition based on constellation diagram,Communication Countermeasures, 1 (2006).

5. The group of Technology and application of electronic war-communication countermeasures, Technology and application of electronic war- communication countermeasures, China, Publishing House of Electronics Industry, 2005: pp. 10-13.

6. S. X. Guo, L. X. Zhang, G. X. Hua, Research on the key techniques in communication countermeasures, Telecommunications Technologies, 6 (2005).

7. M. J. He, Research on sub-set recognition of the MPSK and QAM,Cheng Du: University of Electronic Science and technology, Jan. 2006.

8. C.X. Fan, L. N. Cao, The principle of communication (National Defence Industry Press, 2009).

9. Y. L. Gao, Z. Z. Zhang, Modulation recognition based on combined feature parameter and modified probabilistic neural network, In Proc of the Sixth World Congress on Intelligence Control and Automation, Dalian, China, Jun. 2006, pp. 2954-2958.

10. J. X. Wang, H. Song, Digital modulation recognition based on constellation diagram,Journal of China Institute of Communications, 25 (2004).

11. B. G. MOBASSERI. Digital modulation classification using constellation shape, Signal Processing, 80 (2000).

12. Z. Q. Bian, X. G. Zhang, Pattern recognition, (2nd ed. Tsinghua University press, 1999).

13. A. Swami, B. M. Sadler, Hierarchical digital modulation classification using cumulates,IEEE Trans. Communications, 48 (2000).

14. B. J. Frey, D. Dueck, Clustering by passing messages between data points,Science, 315 (2007). 